\title{
Non-therapeutic (elective) ventilation of potential organ donors: the ethical basis for changing the law
}

\author{
A B Shaw Bradford Hospitals
}

\begin{abstract}
Non-therapeutic ventilation of potential organ donors would increase the supply of kidneys for transplantation. There are no major ethical objections to it. The means of permitting it are forbidden by laws with an ethical basis. A law permitting it would need an ethical basis. Introducing a third legal method of diagnosing death would be unethical. Expanding the power of the advance directive to permit procedures involving minimal harm would be ethical but not helpful. Extending the power of proxies to permit specific nontherapeutic procedures which caused or risked minimal harm to incompetent patients is the best way forward.
\end{abstract}

\section{The current position}

Organs for transplantation are normally obtained from dead patients with the consent of the next of kin. Only the kidney, a paired organ, can come from a live donor. Even here the need in the UK is for 50 kidneys per million people per year but only 30 are available. ${ }^{1}$ Increasing numbers of patients with renal failure are therefore treated by dialysis, which is more expensive than, and inferior to, transplantation. Patients needing hearts, livers or lungs are often denied effective treatment. The supply of organs is insufficient to meet current needs.

Many donor patients have died from a cerebral vascular catastrophe. In some mechanical ventilation has been started because breathing stopped spontaneously before the diagnosis or prognosis was known. When brain stem death occurs the organs are removed for transplantation, with the prior consent of family. The surgery is performed whilst the heart is still supplying oxygenated blood to the organs which therefore remain suitable for transplantation.

In many patients the diagnosis has been made and the prognosis is known to be hopeless before breathing stops. These patients die in emergency departments or general medical wards without being ventilated. The circulation stops and the organs become unsuitable for transplantation.

\section{Key words}

Non-therapeutic ventilation; potential organ donors.

\section{The protocol for non-therapeutic ventilation}

In order to increase the supply of organs patients in Exeter with cerebral catastrophes were ventilated for $N$ non-therapeutic reasons. ${ }^{2}$ The procedure was called elective ventilation but it is better called non-therapeutic, to be quite clear that it is not done for the benefit of the patient. It is analogous to non-therapeutic research.

Patients admitted to general wards or emergency departments with rapidly deepening coma from untreatable intracranial catastrophes were assessed by a senior doctor who decided that death was almost inevitable. The transplant team next decided if the patient was a suitable organ donor. The relatives were then informed of the prognosis and asked if the patient would have wanted to donate organs in the event of death. If there was agreement in principle to organ donation it was explained that death must occur in an intensive care unit so that mechanical ventilation could start as soon as natural breathing stopped in order to keep the kidneys in a state suitable for transplantation. ${ }^{2}$

\section{The result}

The highest rate of organ donation in the United 온 Kingdom was achieved and the number of patients $\frac{7}{O}$ on dialysis in Exeter was kept stable for four years by this method. ${ }^{2}$ The Royal College of Physicians ${ }^{3}$ and $N$ the British Medical Association, ${ }^{4}$ with some qualifications, welcomed the development as useful and ethical.

\section{The objections}

However, lawyers have now advised the Department of Health that this practice is unlawful for two reasons.

Ventilation is started when the patient is not dead. $\mathrm{He} / \mathrm{she}$ is not dead in the classical sense as the heart 2 is still beating and circulating blood. Nor can the brain be declared dead because the tests of brain 8 stem death have not yet been done. It is true that the heart will inevitably stop a few minutes after breathing stops spontaneously unless ventilation is started. 
But until the heart stops or the tests of brain stem death have been done the patient cannot legally be declared dead.

As long as the patient is not legally dead doctors must act solely in the interest of the patient and no proxy has the power to authorise otherwise. ${ }^{5}$ Mechanical ventilation to preserve organs for donation is clearly not performed in the interest of the patient. Indeed prolonging a patient's life by medical interference without his consent when it is not intended in his interest is a civil and criminal wrong. ${ }^{6}$ No doctor may do it and no proxy has authority to consent to it.

The two legal objections are each based on a sound ethical principle. First, medical care must not stop until patients are definitely dead. The definition of death must have a firm biological foundation agreed by the vast majority of the medical establishment and accepted by the public. Second, the interests of living but incompetent people must be protected. The nearly dead are still living. No proxy may be altruistic at their expense as altruistic acts must have the informed consent of the benefactor.

Non-therapeutic ventilation could only be permitted by a new law. It must be shown that the intervention is itself ethical and the law which would allow it is also ethical.

\section{Exceptions to good ethical rules}

Hare proposes certain steps be followed when considering whether to permit a new practice which breaches an established ethical rule. ${ }^{7} \mathrm{He}$ is a utilitarian philosopher always at pains to respect established "deontological" rules and practices where doing so can be justified. I have adapted his approach as follows.

1. The new practice must bring substantial benefits unobtainable by other means.

2. We must clarify precisely why following the established rule is beneficial and abandoning it would be detrimental.

3 . These reasons must not apply to the proposed exception.

4. The exception must be clearly defined so there is no slippery slope leading to a general abandonment of the established rule.

5. The new practice must not itself cause serious new harms nor flout other important ethical rules.

\section{Ways of increasing the supply of donor organs}

There would be more cadaver organs for transplantation if more people carried donor cards and registered their wish to donate. Fewer relatives would refuse donation if they were approached by specially trained nurses. ${ }^{8}$ Some countries adopt an opting-out rather than an opting-in policy. The organs are taken unless a prior refusal has been registered by the patient. Most believe this is an unacceptable assault on the autonomy of potential donors and their families. More kidneys might be taken from related live donors. However, analysis suggests that these approaches would have a limited effect. ${ }^{9}$

The problem may one day be solved by using organs from transgenic pigs. Meanwhile the waiting list for donor kidneys increases because of two desirable developments: there are fewer fatal head injuries and medical advances have enabled more patients to benefit from transplantation. ${ }^{9}$

Therefore non-therapeutic ventilation is necessary to increase the supply of donor organs. The ethical and practical objections to it must be considered.

\section{Specific objections to non-therapeutic ventilation}

A patient might enter the persistent vegetative state. ${ }^{10}$ According to the Exeter experience this would occur rarely. ${ }^{2}$ If it did not the permissibility of the practice would require review. It must be emphasised that the distress caused by such an unfortunate event would affect the family which had knowingly accepted the risk and not the insentient patient. In the current state of the law judicial permission would be required to discontinue ventilation after a year. ${ }^{6}$ If such an event resulted in a claim for damages it is likely that the cost of a settlement would be considerably smaller than the total saving to the NHS.

A slippery slope might begin when the nearly dead were treated in the way we now treat the really dead. One could remove patients dying from cancer from the ward when they slip into coma and use the bed for the ill patient waiting in casualty. Here also the dying patient loses nothing and the living one gains. However, the permission for ventilation would be statutory and specific. The consent of family would be necessary and the supervision would be close, in the interest both of the family and of the patient.

Death in hospital today is often associated with technical interventions which reduce the dignity of the dying process. Ventilation of a patient with no hope of recovery prolongs dying and is a further physical indignity. However, the moral dignity of death is increased when it directly benefits others. Families should be free to venerate their dead in their own way. To some it means leaving the body intact. To some it would mean making sense of an untimely death by ensuring that at least some good resulted.

Relatives might later regret having given consent. ${ }^{11}$ Equally they might be pleased. The objection applies to all cadaver organ donation. We need studies to determine whether relatives were satisfied a few years later with the decisions they had made. 
Moving the dying patient to intensive care would use the scarce resource of intensive care beds for dead patients. The extra bed-days needed would be less than one per cent but kidney transplants might increase by more than 50 per cent. ${ }^{9}$ Over five years dialysis costs three times as much as transplantation. The money saved could be used to provide more intensive care beds. İt would be sad if the National Health "business" had become so ossified and commercial that such a transfer of funds would be impossible.

It might demoralise intensive care staff already under intense pressure ${ }^{11}$ : they treat patients for life not for death. However, all intensive care units are obliged to continue for a period the ventilation of patients for whom all real hope has been abandoned. The number of potential donors in each unit would be few. Education and support would minimise the harm to staff.

A situation might arise in which a potential donor was accepted by the intensive care unit when there was space but later there was no bed left for another patient. No patient should be deprived of therapeutic ventilation so that another could be given nontherapeutic ventilation to benefit a third. It would have to be explained to relatives that the donation would not take place if another patient required ventilation.

It might cause public concern that dying patients were being used as organ fodder. However, it could not be done without the consent of the family. Many new procedures arouse public concern. If the procedure is beneficial and ethical this is best dealt with by public discussion of the benefits and risks and the safeguards against abuse.

All medical advances create some problems but there are no major ethical objections to this beneficial intervention. Is there then an ethical way to legalise it?

\section{The diagnosis of death}

Riad and Nicholls claim that death actually occurs when the patient stops breathing, even though the formal diagnosis is made later. ${ }^{12}$ If this were so, proxy consent to organ donation would be permissible as with other cadavers. What then is the ethical basis of the current legal definition of death?

When breathing stops the heart stops within a few minutes because of oxygen lack. Inevitably the brain quickly dies. Therefore death was always diagnosed when the breathing and the circulation stopped and still is. After a cerebral catastrophe breathing stops because brain stem function is lost. If the patient is ventilated mechanically the heart continues to beat automatically, the patient stays warm and excretes urine. But once the brain stem is dead no higher part of the brain will ever function again. The patient will never breathe again nor emerge from the deepest coma. Eventually the heart stops despite continued ventilation.

Therefore mechanical ventilation has made us realise that death is really the certain, total, and irreversible loss of organised brain function, even though the heart still beats.

Tests of brain stem death have therefore been developed so that corpses do not have to be ventilated. ${ }^{13}$ It may be diagnosed if, on two occasions separated by an interval, removal from the ventilator and exposure to carbon dioxide does not stimulate respiration and intense stimulation of brain stem reflexes does not produce a response. After diagnosis mechanical ventilation may be stopped, the heart soon stops and the patient may be removed from the ward.

A secondary benefit is that the organs of the patient can be used for transplantation. In this case ventilation continues until surgery has been performed.

\section{A third method of diagnosing death}

If the patient is ventilated when the breathing stops spontaneously after a cerebral catastrophe the otherwise inevitable death cannot be diagnosed by legally accepted criteria. The heart is still beating. Brain stem death tests have not been done.

As death would be inevitable without ventilation could a third legal definition of death have an ethical basis? Could death be diagnosed when breathing stops after a cerebral catastrophe in a deeply comatose patient for whom mechanical ventilation is not indicated?

Only a certainly irreversible state can be called death. All nine patients treated in Exeter suffered brain stem death within 24 hours. ${ }^{2}$ However, until there has been extensive experience of non-therapeutic ventilation we cannot say this will always happen. Certainly patients who require ventilation after neurosurgery recover. Swelling of the brain might improve during mechanical ventilation. An occasional patient might start to breathe again even if only to enter a persistent vegetative state. ${ }^{10}$ Good ethics must be based on truth. As there is a risk that non-therapeutic ventilation has arrested the dying process the patients cannot be treated as dead.

We cannot accumulate the experience necessary to show that potential donors always suffer brain stem death despite ventilation because it is at present illegal to carry out non-therapeutic research on incompetent patients. It is not always unethical and certainly not so unethical that we should refuse to use the knowledge gained if the research were done elsewhere. However, even if all these patients soon suffered brain stem death problems would remain.

The two current legal ways of diagnosing death are based entirely on the interest of the patient. They ensure that when we are dead we are treated as such but not until then. To introduce a third definition of death in the interest not of the patient but of another 
person would be unethical. The Helsinki declaration states that the interests of the patient must take precedence over those of society. The nearly dead must not be treated as really dead. There is the danger of a slippery slope. If simple cessation of breathing in the presence of a heartbeat were accepted as evidence of death in one circumstance, it might be in others, such as in terminal cancer. There would be a risk that such a patient would not be dead and might even breathe again.

We define death very tightly because we must never abandon living patients, never act against their interests, nor use them just as a means to achieve another purpose. It is vital that all patients are confident that this is so. The concept of brain stem death achieved acceptance by the medical establishment and the public with some difficulty. It is still challenged by some philosophers. ${ }^{14}$ Legalising a third definition of death in the interest of other patients would arouse opposition and anxiety. There is a fear that live people might be used as a source of organs. There was a cartoon of a patient with the notice "Asleep, not a heart donor" pinned to his chest. More relatives, not fewer, might refuse donation.

Permitting non-therapeutic ventilation by this method would breach the third, fourth and fifth rules given above.

\section{Consent by advance directive}

It is ethical for people voluntarily to put the interests of others before those of their own health. Blood donation, marrow donation, and kidney donation by living relatives are examples. Kidney donation even by unrelated people can be authorised by the Unrelated Live Transplant Regulatory Authority. ${ }^{15}$ The concept of the patient's interests is rightly enlarged to include the psychological and moral benefits of altruism. Consent must be informed and free. The damage suffered must be small compared with the benefit conferred. Mechanisms must always be in place to protect people from inappropriate harm through zeal or ignorance.

Non-therapeutic ventilation meets these criteria and there is no ethical reason why informed consent to it should not be given by advance directive.

The formula for a donor card might be: "In the event of my impending inevitable death from a severe stroke I would like to be moved to an intensive care unit. I would wish all supportive medical care to be given to me until I stop breathing spontaneously. When that happens I wish to have my organs kept in good condition by mechanical ventilation so that they can be transplanted to a patient in need of them as soon as the diagnosis of brain death is confirmed."

It would not be difficult to permit doctors to comply with advance directives to perform specific non-therapeutic procedures which did minimal harm and were important to the values of an incompetent patient. ${ }^{16}$ Advance directives instructing a doctor to refrain from prolonging life of no value to the patient are legally valid. ${ }^{17}$ So are advance directives which continue refusal of a specific clinically beneficial intervention after the patient has become incompetent. ${ }^{18}$ The duty to respect autonomy trumps the duty of beneficence. It would be entirely ethical for advance directives permitting non-therapeutic ventilation to have legal force.

Unfortunately it would not often help. About 20 per cent of the public carry a donor card on their person. ${ }^{9}$ Few of us discuss death with our relatives even though transplantation from cadavers has been practised for many years. Children are no longer brought up to be familiar with death. We are reluctant to contemplate it or discuss it with those near to us when the need to do so is not obvious. It is unlikely that donor cards will be more widely carried. What is worse, is that it might hinder. It is hard enough to get the public to carry cards indicating a wish to donate organs after death. It might be disastrous to run another campaign asking people to sign up for organ donation before death.

This approach fails on the last of the ethical criteria stated. New problems would be caused.

\section{Consent by proxy}

We have a duty to obtain the informed consent of a competent person before a beneficial clinical intervention. It is far more important to obtain consent to an intervention which is not beneficial. Therefore proxies can consent only to interventions in the interest of an incompetent person.

In practice there are exceptions. Parents give consent to non-therapeutic research on their children provided both risk and harm are minimal. ${ }^{19}$ With similar restrictions ethics committees allow research on patients unable to give consent because of dementia or critical illness. ${ }^{20} 21$ The justification in each case is that the harm is minimal, the benefit can be obtained no other way, and there is a belief that the patient would have consented if competent.

In Britain young siblings are used as marrow donors. Here the organ can regenerate although the procedure requires a general anaesthetic. In France, with important safeguards, a kidney, which does not regenerate, may be removed from a minor although few other countries would allow this. ${ }^{15}$

Non-therapeutic ventilation causes minimal harm. Delaying death for a short time by keeping someone in a deeply comatose state on a ventilator causes no distress to the patient himself. If the family, from their knowledge of the patient, believe that organ donation would have been desired, permitting the minimal harm entailed is ethical.

In British law only the interest of the incompetent person is considered unless he has clearly stated his wish when competent. It has tended to keep to a 
narrow definition of interest. Permission to stop feeding patients in the persistent vegetative state has been granted, not because it is what the patient would have wanted, but because feeding was not in the interest of the patient. ${ }^{6}$ American law considers substituted judgment as well. The broader view is that doing what we believe incompetent persons would have wanted, and acting in accordance with their known values, also serves their interest. It does not further the interest of a child if it later regrets not having been allowed to make a tissue donation to a sibling. A dead person may never regret not being used as an organ donor. But we all want our lives to have meaning at the end as well as throughout.

The Law Commission report recognises this and proposes in a draft bill to permit three types of intervention which would benefit others and not the incompetent person. ${ }^{22}$ These are non-therapeutic research, non-therapeutic ventilation, and the removal of material for genetic studies. The emphasis in the report is on the protection of the incompetent person. However, it is also important that the family of the incompetent person should be protected.

In the case of ventilation the patient should be protected by a senior doctor, probably a neurologist, not involved with either the care of the patient or the donation. The family and the patient should also be protected by a trained social worker or psychologist, rather than a lawyer or administrator, as suggested by the commission.

Discussion with the family is a sensitive and skilled matter. It is essential that the consent of family members is freely given and that they arrive at the decision which they will think in later years was right for them. In the interest of the patient the family should only give consent if they have reason to believe that the patient would have done so.

Consent would be given by the same relatives as now give consent to cadaver donation. It might or might not be better to define this legally.

Legalising non-therapeutic research on the incompetent would also be tightly controlled. The patient would be protected by research ethics committees and the family by an independent adviser. Breaching guidelines would be serious professional misconduct and new statutory bodies and legal penalties would not be necessary to avoid improper ventilation or research.

Hare's criteria are fulfilled. Important benefits otherwise unobtainable are achieved. Death is still defined in the interest of the patient alone. The principle of protecting the incompetent person is intact. The non-therapeutic interventions permitted are specific and supervised so there is no slippery slope. Detriments associated with the new practice would be less than the benefits. There are no serious new ethical problems. It would be ethical to change the law to allow proxies to permit non-therapeutic ventilation of potential donors.

\section{The way forward}

Improvements in medical practice are carefully studied by a series of trials before general adoption. This does not occur with changes in the law or politically motivated administrative change. There is a need for a legal mechanism for permitting an adequate trial of the Exeter protocol. Without one we either stifle a promising development or give it universal acceptance before its consequences are fully known. Nevertheless in this situation proxies should be allowed to consent to non-therapeutic ventilation. There are no major ethical obstacles. The prospective benefits are much greater than the detriments.

Dr Allen B Shaw, MD, FRCP, is Consultant Physician at St Luke's Hospital, Bradford, West Yorkshire.

\section{References}

1 United Kingdom Transplant Support Service Authority (UKTSSA). Annual report 1988/89. Bristol: UKTSSA, 1989.

2 Feest TG, Riad HN, Collins CH, Golly MG, Nicholls A J, Hamad SN. Protocol for increasing organ donation after cerebrovascular deaths in a district general hospital. Lancet 1990; 335: 1133-5.

3 Williams R. More donor organs for transplantation. Elective ventilation proposals. Fournal of the Royal College of Physicians of London 1993; 27: 214-5.

4 Somerville A. Medical ethics today: its practice and philosophy. London: BMJ Publishing Group, 1993: 27-9.

5 Jones MA. Elective ventilation of potential organ donors. The legal background. British Medical fournal 1995; 310: 717-8.

6 Browne-Wilkinson LJ. Airedale NHS Trust $\mathrm{v}$ Bland [1993] All ER 821.

7 Hare RM. In vitro fertilisation and the Warnock report. In: Chadwick RF, ed. Ethics, reproduction and genetic control. London: Routledge, 1994: 63-90.

8 Dorozynski A. French GPs launch organ donor campaign. British Medical fournal 1995; 311: 214-5.

9 New B, Solomon M, Dingwall R, McHale J. A question of give and take. Improving the supply of donor organs for transplantation. King's Fund Institute: research report 18, 1994.

10 Routh G. Elective ventilation for organ donation - the case against. Care of the Critically Ill 1992; 8: 61-2.

11 Neuberger J. Elective ventilation of potential organ donors. The lay view. British Medical fournal 1995; 310: 715-6.

$12 \mathrm{Riad} \mathrm{H}$, Nicholls A. Elective ventilation of potential organ donors. British Medical fournal 1995; 310: 714-5.

13 Conference of medical royal colleges and their faculties (UK). Diagnosis of death. British Medical fournal 1976; 2: 1187-9.

14 Evans M. Against brainstem death. In: Gillon R, ed. Principles of health care ethics. Chichester: John Wiley and Sons, 1994.

15 Mason JK. Organ donation and transplantation. In: Dyer C, ed. Doctors, patients and the law. Oxford: Blackwell Scientific Publications, 1992.

16 Francis R. Elective ventilation of potential organ 
donors. A legal comment. British Medical fournal 1995; 310: 718.

17 McLean SAM. Making advance medical decisions. Fournal of the Medical and Dental Defence Union 1995; 11: 28-9.

$18 \mathrm{Re} \mathrm{C}$ (adult refusal of medical treatment) [1994] 1 All ER 819.

19 British Paediatric Association. Guidelines for the ethical conduct of medical research involving children. London:
British Paediatric Association, 1992.

20 Royal College of Physicians of London. Research involving patients. London: Royal College of Physicians of London, 1990.

21 Royal College of Psychiatrists. Guidelines for research ethics committees. Psychiatric Bulletin 1990; 14: 48-61.

22 Law Commission Report 231. London: Law Commission, 1995.

\section{Correction}

In the paper, "Mummy was a fetus: motherhood and fetal ovarian transplantation", 1995; 21 : 298-304, the number of abortions in the United States for 1988 was given as 58,192 . The correct number of abortions in the United States for that year is 1.59 million. 